\title{
COMMENT
}

\section{Magnesium sulfate: a last roll of the dice for anti-excitotoxicity?}

\author{
Robert Galinsky ${ }^{1}$, Laura Bennet ${ }^{1}$ and Alistair J. Gunn (D) ${ }^{1}$ \\ Pediatric Research (2019) 86:685-687; https://doi.org/10.1038/s41390-019-0539-9
}

\begin{abstract}
Therapeutic hypothermia for neonatal encephalopathy (NE) has successfully translated to standard care, confirming the fundamental validity of extensive preclinical research across multiple species and settings. ${ }^{1}$ The challenge now is to further improve neuroprotection, so that more babies survive NE without disability. Current protocols for therapeutic hypothermia appear to be near-optimal, ${ }^{2-4}$ so the obvious solution is to add other neuroprotective agents to hypothermia. Logically, we should follow the example of the development of anticancer drugs and systematically test different combinations in a stepwise fashion, starting with agents that are already clinically approved for other indications. ${ }^{5}$
\end{abstract}

\section{EXCITOTOXICITY DURING HYPOXIA-ISCHEMIA (HI)}

Early research on the mechanisms of delayed cell death after $\mathrm{HI}$ focused on the observation that excitatory amino acids ("excitotoxins") such as glutamate accumulate in the extracellular space during $\mathrm{HI}$ and during intense seizures. ${ }^{6}$ Exposure to excitotoxins can facilitate excessive entry of calcium into the cell and so activate delayed cell death pathways. ${ }^{5}$ There was great excitement when specific antagonists seemed to dramatically reduce cell death. ${ }^{7}$ We now know that these excitatory channels are just one of many calcium channels that open during $\mathrm{HI}^{8}$ and that the apparent benefit in small animal studies was confounded by drug-induced hypothermia. ${ }^{7}$ Consequently, excitotoxin antagonists failed to translate in adult clinical trials. ${ }^{9}$ Further, in preterm fetal sheep, infusion of the non-competitive glutamatergic antagonist dizocilpine after $\mathrm{HI}$ was associated with very limited improvement in neuronal survival, in only one hippocampal region, and combined dizocilpine infusion with hypothermia did not augment hypothermic neuroprotection. $^{10}$

\section{COULD INCREASED SERUM MAGNESIUM AUGMENT HYPOTHERMIC NEUROPROTECTION?}

Magnesium is an endogenous, physiological anti-excitotoxic agent, acting by voltage-dependent inhibition of glutaminergic channels. ${ }^{11,12}$ There are multiple pragmatic features in its favor as a potential neuroprotectant, as well as encouraging preclinical and clinical evidence. It is very inexpensive, its physiological effects are relatively well understood, ${ }^{13,14}$ including vasodilation at high concentrations, and it is widely used in clinical practice and so regulatory approval would be easily gained. Systematic metaanalysis of five randomized controlled trials of magnesium sulfate
$\left(\mathrm{MgSO}_{4}\right)$ in NE suggested that it significantly reduced adverse short-term outcomes, with trends for improved long-term outcomes but greater mortality. ${ }^{15}$ Critically, meta-analysis of large randomized controlled trials of maternal $\mathrm{MgSO}_{4}$ in preterm labor found that it was associated with significantly reduced risk of cerebral palsy (number needed to treat: 64$).{ }^{16}$ The pathophysiological basis of this association is unclear, and the effects on the primary outcome of death or disability were mixed; a significant overall effect was seen only in the subset of trials designed to test for neuroprotection.

The preclinical evidence for neuroprotection after $\mathrm{HI}$ with $\mathrm{MgSO}_{4}$ is mixed. In rodents, initial studies of treatment after $\mathrm{HI}$ or ischemia were very promising. ${ }^{17}$ These studies did not control for the vasodilator effects of magnesium that can promote confounding hypothermia. Studies that included rigorous temperature control showed little benefit. ${ }^{17} \mathrm{~A}$ recent study showed dramatic protection with $\mathrm{MgSO}_{4}$ given before $\mathrm{HI}$ in rats. ${ }^{18}$ However, $\mathrm{MgSO}_{4}$ was given as large boluses before $\mathrm{HI}$, and the evidence suggests that protection was mediated through preconditioning. This would not be practical after $\mathrm{HI}$ injury. In large animals, $\mathrm{MgSO}_{4}$ by itself was not neuroprotective after $\mathrm{HI}$ in piglets $^{19,20}$ or in near-term fetal sheep. ${ }^{21}$ Indeed, in preterm fetal sheep $\mathrm{MgSO}_{4}$ infusion for $48 \mathrm{~h}$ after acute $\mathrm{HI}$ was associated with impaired oligodendrocyte maturation. ${ }^{22}$

Notwithstanding these results, in adult rodents, studies of combined $\mathrm{MgSO}_{4}+$ hypothermia after ischemia suggest additive benefit. ${ }^{23-25}$ Moreover, $\mathrm{MgSO}_{4}$ has significant antiseizure properties. It has been used for many years to reduce the risk of maternal seizures during moderate-to-severe eclampsia and may be more effective than anticonvulsants, such as phenytoin. ${ }^{26}$ Consistent with this, in preterm fetal sheep, $\mathrm{MgSO}_{4}$ infusion markedly reduced seizures after profound $\mathrm{HI}$, with greater benefit seen in male fetuses. ${ }^{27}$ Seizures are associated with increased anaerobic stress in the brain in neonatal NE. ${ }^{28}$ In normothermic near-term fetal sheep, abolishing post-ischemic seizures with an infusion of dizocilpine reduced injury in more mildly affected regions, although not in the core infarct. ${ }^{29}$ Given that therapeutic hypothermia reduces but does not abolish seizures during $\mathrm{NE}^{30,31}$ it is plausible that combining hypothermia and Mg could further suppress seizures and associated excitotoxic stress and so improve outcomes. This background strongly suggests that $\mathrm{MgSO}_{4}+$ hypothermia combination therapy is well worth testing despite its limitations as a sole therapy. Given that safety trials of hypothermia plus $\mathrm{MgSO}_{4}$ have already being undertaken, ${ }^{32-34}$ it was urgent to undertake rigorous preclinical studies in large animal, translational models.

${ }^{1}$ The Department of Physiology, The University of Auckland, Auckland, New Zealand

Correspondence: Alistair J. Gunn (aj.gunn@auckland.ac.nz)

Received: 24 July 2019 Accepted: 2 August 2019

Published online: 14 August 2019 


\section{MAGNESIUM SULFATE PLUS HYPOTHERMIA IN THE PIGLET}

In this issue, Lingam et al. report that the combination of an infusion of $\mathrm{MgSO}_{4}$ with hypothermia after $\mathrm{HI}$ in anesthetized male, term piglets was safe and was associated with a small, incremental improvement in some but not all endpoints. ${ }^{35}$ Encouragingly, after $48 \mathrm{~h}$ recovery, compared to hypothermia alone the combination was associated with a small reduction in the total number of dead brain cells, summed across all brain regions, and increased numbers of surviving oligodendrocytes in the white matter tracts, although myelination itself was not assessed. However, there was no significant improvement in cell death in any brain region taken in isolation. Further, there was no significant improvement in recovery of amplitude integrate electrophysiological (aEEG) activity or in magnetic resonance spectroscopy (MRS) parameters that clinically are closely associated with neurodevelopmental outcomes at 18 months of age after NE. ${ }^{36}$ Although the authors report that there was a trend toward better recovery of aEEG $(p=$ 0.09 ), it is of concern that the authors also found an increase in activated caspase- $3 .^{35}$ This raises the possibility that there was upregulation of cell death that might attenuate the apparent histological benefit during a longer period of recovery. Finally, the reader should note that treatment was started just $1 \mathrm{~h}$ after $\mathrm{HI}$. It is very challenging to start an experimental intervention this soon after birth. Given that essentially all neuroprotective treatments show dramatic loss of efficacy with greater delay after $\mathrm{HI}^{1}{ }^{1}$ it is likely that the results of the current study represent the best possible outcome for this approach.

\section{CONCLUSION}

Although it is not what was hoped for, the present findings from Lingam et al. are highly valuable negative information. Critically, the lack of benefit on the clinically well-validated MRS measures of outcome suggests that we should not proceed to a phase three clinical trial of add-on $\mathrm{MgSO}_{4}$ for NE. This is an important saving of time, money, and effort. In the longer term, given its excellent safety profile, it is just plausible that it might be considered for studies of multidrug "cocktails" for neuroprotection, but much more work is vital before such a complex study can be considered. Finally, these data provide further evidence that anti-excitotoxic therapy after $\mathrm{HI}$ has limited benefit for the developing brain.

\section{ACKNOWLEDGEMENTS}

This work was supported by the Health Research Council of New Zealand (17/601) and (16/003) and the NHMRC CJ Martin Fellowship (1090890).

\section{AUTHOR CONTRIBUTIONS}

All authors contributed to the conception and design of the manuscript, drafting the article, and approving the final version.

\section{ADDITIONAL INFORMATION}

Competing interests: The authors declare no competing interests.

Publisher's note: Springer Nature remains neutral with regard to jurisdictional claims in published maps and institutional affiliations.

\section{REFERENCES}

1. Gunn, A. J. et al. Therapeutic hypothermia translates from ancient history in to practice. Pediatr. Res. 81, 202-209 (2017).

2. Zhou K. Q., et al. Protection of axonal integrity with 48 or $72 \mathrm{~h}$ of cerebral hypothermia in near-term fetal sheep. Pediatr. Res. https://doi.org/10.1038/ s41390-019-0475-8 (2019).

3. Davidson, J. O. et al. How long is sufficient for optimal neuroprotection with cerebral cooling after ischemia in fetal sheep? J. Cereb. Blood Flow Metab. 38, 1047-1059 (2018)
4. Shankaran, S. et al. Effect of depth and duration of cooling on death or disability at age 18 months among neonates with hypoxic-ischemic encephalopathy: a randomized clinical trial. JAMA 318, 57-67 (2017).

5. Ferriero, D. M. Neonatal brain injury. N. Engl. J. Med. 351, 1985-1995 (2004)

6. Tan, W. K. et al. Accumulation of cytotoxins during the development of seizures and edema after hypoxic-ischemic injury in late gestation fetal sheep. Pediatr. Res. 39, 791-797 (1996).

7. DeBow, S. B., Clark, D. L., MacLellan, C. L. \& Colbourne, F. Incomplete assessment of experimental cytoprotectants in rodent ischemia studies. Can. J. Neurol. Sci. 30, 368-374 (2003).

8. Cross, J. L., Meloni, B. P., Bakker, A. J., Lee, S. \& Knuckey, N. W. Modes of neuronal calcium entry and homeostasis following cerebral ischemia. Stroke Res. Treat. 2010, 316862 (2010).

9. Gorelick, P. B. Neuroprotection in acute ischaemic stroke: a tale of for whom the bell tolls? Lancet 355, 1925-1926 (2000).

10. George, S. A. et al. Nonadditive neuroprotection with early glutamate receptor blockade and delayed hypothermia after asphyxia in preterm fetal sheep. Stroke 43, 3114-3117 (2012).

11. Traynelis, S. F. et al. Glutamate receptor ion channels: structure, regulation, and function. Pharm. Rev. 62, 405-496 (2010).

12. Hallak, M., Hotra, J. W., Custodio, D. \& Kruger, M. L. Magnesium prevents seizureinduced reduction in excitatory amino acid receptor (kainate and alpha-amino-3hydroxy-5-methylisoxazole-4-propionic acid) binding in pregnant rat brain. Am. J. Obstet. Gynecol. 183, 793-798 (2000).

13. Galinsky, R. et al. Magnesium sulfate and sex differences in cardiovascular and neural adaptations during normoxia and asphyxia in preterm fetal sheep. Am. J. Physiol. Regul. Integr. Comp. Physiol. 315, R205-R217 (2018).

14. Galinsky, R. et al. Magnesium sulphate and cardiovascular and cerebrovascular adaptations to asphyxia in preterm fetal sheep. J. Physiol. 594, 1281-1293 (2016).

15. Tagin, M., Shah, P. S. \& Lee, K. S. Magnesium for newborns with hypoxic-ischemic encephalopathy: a systematic review and meta-analysis. J. Perinatol. 33, 663-669 (2013).

16. Doyle, L. W., Crowther, C. A., Middleton, P., Marret, S. \& Rouse, D. Magnesium sulphate for women at risk of preterm birth for neuroprotection of the fetus. Cochrane Database Syst. Rev. CD004661 (2009).

17. Galinsky, R. et al. Magnesium is not consistently neuroprotective for perinatal hypoxia-ischemia in term-equivalent models in preclinical studies: a systematic review. Dev. Neurosci. 36, 73-82 (2014).

18. Koning, $\mathrm{G}$. et al. Magnesium induces preconditioning of the neonatal brain via profound mitochondrial protection. J. Cereb. Blood Flow Metab. 39, 1038-1055 (2019).

19. Penrice, J. et al. Magnesium sulfate after transient hypoxia-ischemia fails to prevent delayed cerebral energy failure in the newborn piglet. Pediatr. Res. 41, 443-447 (1997).

20. Greenwood, K. et al. Magnesium sulfate treatment after transient hypoxiaischemia in the newborn piglet does not protect against cerebral damage. Pediatr. Res. 48, 346-350 (2000).

21. de Haan, H. H., Gunn, A. J., Williams, C. E., Heymann, M. A. \& Gluckman, P. D. Magnesium sulfate therapy during asphyxia in near-term fetal lambs does not compromise the fetus but does not reduce cerebral injury. Am. J. Obstet. Gynecol. 176, 18-27 (1997)

22. Galinsky, R. et al. Magnesium sulfate reduces EEG activity but is not neuroprotective after asphyxia in preterm fetal sheep. J. Cereb. Blood Flow Metab. 37, 1362-1373 (2017)

23. Campbell, K., Meloni, B. P. \& Knuckey, N. W. Combined magnesium and mild hypothermia ( 35 degrees $C$ ) treatment reduces infarct volumes after permanent middle cerebral artery occlusion in the rat at 2 and 4, but not $6 \mathrm{~h}$. Brain Res. 1230, 258-264 (2008).

24. Zhu, H., Meloni, B. P., Bojarski, C., Knuckey, M. W. \& Knuckey, N. W. Post-ischemic modest hypothermia ( 35 degrees $C$ ) combined with intravenous magnesium is more effective at reducing CA1 neuronal death than either treatment used alone following global cerebral ischemia in rats. Exp. Neurol. 193, 361-368 (2005).

25. Zhu, H., Meloni, B. P., Moore, S. R., Majda, B. T. \& Knuckey, N. W. Intravenous administration of magnesium is only neuroprotective following transient global ischemia when present with post-ischemic mild hypothermia. Brain Res. 1014, 53-60 (2004).

26. Duley, L., Henderson-Smart, D. J. \& Chou D. Magnesium sulphate versus phenytoin for eclampsia. Cochrane Database Syst. Rev. CD000128 (2010).

27. Bennet, L. et al. Time and sex dependent effects of magnesium sulphate on postasphyxial seizures in preterm fetal sheep. J. Physiol. 596, 6079-6092 (2018).

28. Miller, S. P. et al. Seizure-associated brain injury in term newborns with perinatal asphyxia. Neurology 58, 542-548 (2002).

29. Tan, W. K., Williams, C. E., Gunn, A. J., Mallard, C. E. \& Gluckman, P. D. Suppression of postischemic epileptiform activity with MK-801 improves neural outcome in fetal sheep. Ann. Neurol. 32, 677-682 (1992). 
Magnesium sulfate: a last roll of the dice for anti-excitotoxicity? R Galinsky et al.

30. Low, E. et al. Cooling and seizure burden in term neonates: an observational study. Arch. Dis. Child. Fetal Neonatal Ed. 97, F267-F272 (2012).

31. Harbert, M. J. et al. Hypothermia is correlated with seizure absence in perinatal stroke. J. Child Neurol. 26, 1126-1130 (2011).

32. Nonomura, M. et al. Combination therapy with erythropoietin, magnesium sulfate and hypothermia for hypoxic-ischemic encephalopathy: an open-label pilot study to assess the safety and feasibility. BMC Pediatr. 19, 13 (2019).

33. Ur Rahman, S. et al. Multicenter randomized controlled trial of therapeutic hypothermia plus magnesium sulfate versus therapeutic hypothermia plus placebo in the management of term and near-term infants with hypoxic ischemic encephalopathy (The Mag Cool study): a pilot study. J. Clin. Neonatol. 4, 158-163 (2015).

34. Gulczynska, E. et al. Feasibility and safety of combining therapeutic hypothermia with magnesium sulfate administration, in the management of neonates with hypoxic ischemic encephalopathy - randomized control trial. Neonatal Pediatr. Med. 4, 165 (2018).

35. Lingam, I. et al. Short-term effects of early initiation of magnesium Infusion combined with cooling after hypoxia-ischemia in term piglets. Pediatr. Res. https://doi.org/10.1038/s41390-019-0511-8 (2019).

36. Thayyil, S. et al. Cerebral magnetic resonance biomarkers in neonatal encephalopathy: a meta-analysis. Pediatrics 125, e382-e395 (2010). 